Sir,

\section{Posterior vitreous cyst and intermediate uveitis}

Vitreous cysts are rare structural entities that have been reported sporadically in the literature. Several associations have been described, including retinitis pigmentosa, Toxoplasmosis, trauma, chorioretinal atrophy, and Wagner disease. ${ }^{1}$ We present the first report of a vitreous cyst attached to the peripheral retina in a patient with intermediate uveitis.

\section{Case report}

A 31-year-old woman presented with a 3-day history of floaters in her left eye. There was no past medical or ophthalmic history of note. On examination, her visual

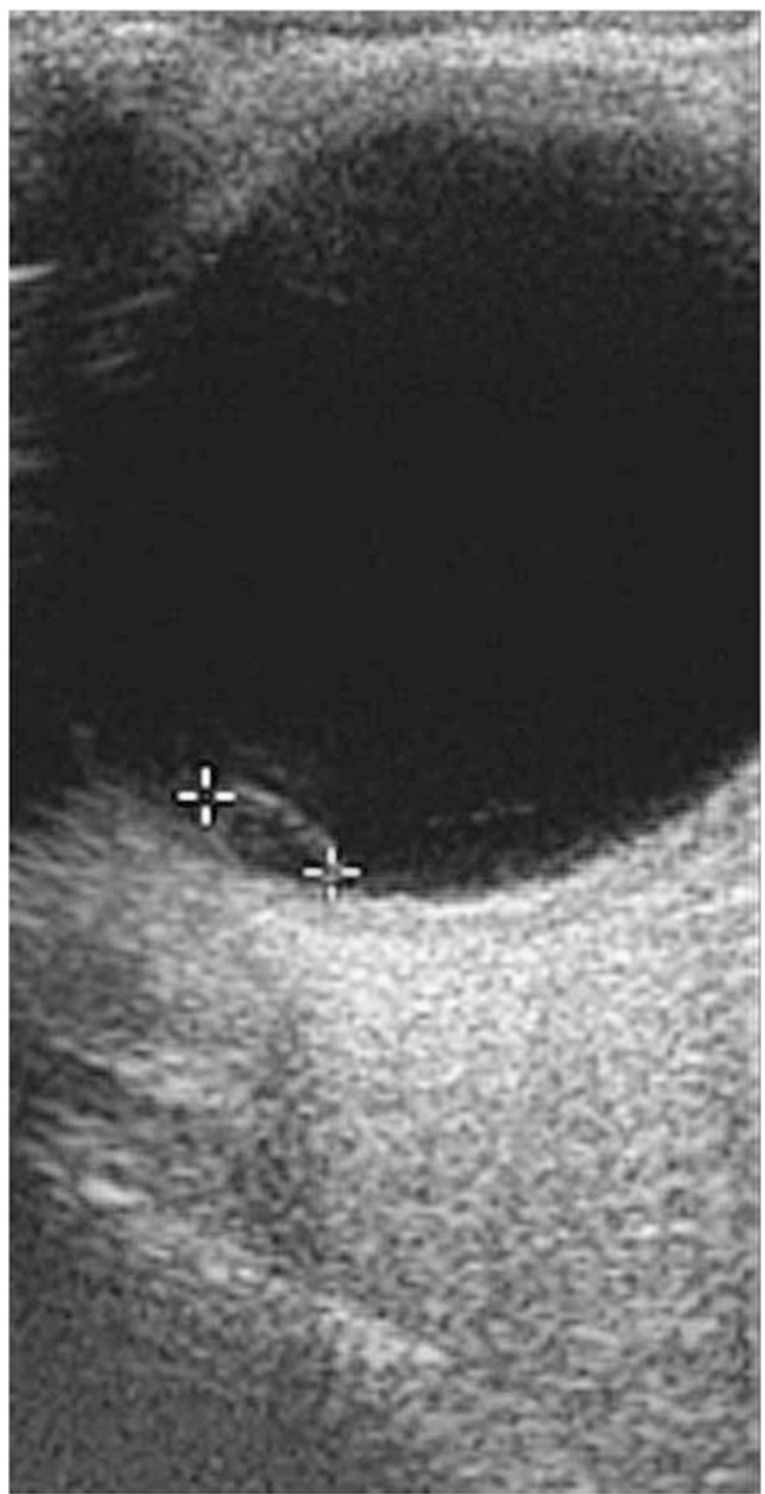

Figure 1 B-scan ultrasound of the vitreous cyst attached to the retina. acuity was $6 / 5$ bilaterally. Her anterior segments were unremarkable and fundoscopy revealed mild vitritis and vitreous snowballs along with peripheral vascular sheathing on the right. In addition, a $4 \mathrm{~mm}$ vitreous cyst attached to the superior equatorial retina was observed (Figure 1). The cyst was adjacent to an area of vascular sheathing and there was evidence of white snowballs on its wall (Figure 2). Vitreous and retina in the fellow eye were unremarkable.

The patient was managed by observation, and 3 months later her symptoms had significantly improved with reduction of the vitreous activity. The vitreous cyst remained unchanged regarding its size, location, and clinical characteristics.

\section{Comment}

Although vitreous cysts have been reported more than a century ago, their pathogenesis remains unclear. They can be congenital or acquired, pigmented or not pigmented, and are usually free floating. ${ }^{1}$

Vitreous cysts in association with uveitis are extremely rare. Brewerton reported the presence of two vitreous cysts in a patient with irido-cyclitis. ${ }^{2}$ More recently, vitreous cysts have been observed in association with Toxoplasmosis and Nematode endophthalmitis. ${ }^{3,4}$

This is the first report of a posterior vitreous cyst attached to the retina in a patient with intermediate uveitis. Vitreous manifestations in intermediate uveitis include vitreitis, aggregates of white exudates analogous to snowballs, and snowbank. ${ }^{5}$ The vitreous cyst in our case may be a coincidental finding or may represent the result of vitreous degenerative changes following chronic irritation from the adjacent inflamed retina. We could also speculate that the vitreous cyst may constitute a rare transformation of previous large or coalesced snowballs.

Clinicians should be aware of the possibility of vitreous cysts developing in patients with intermediate uveitis.

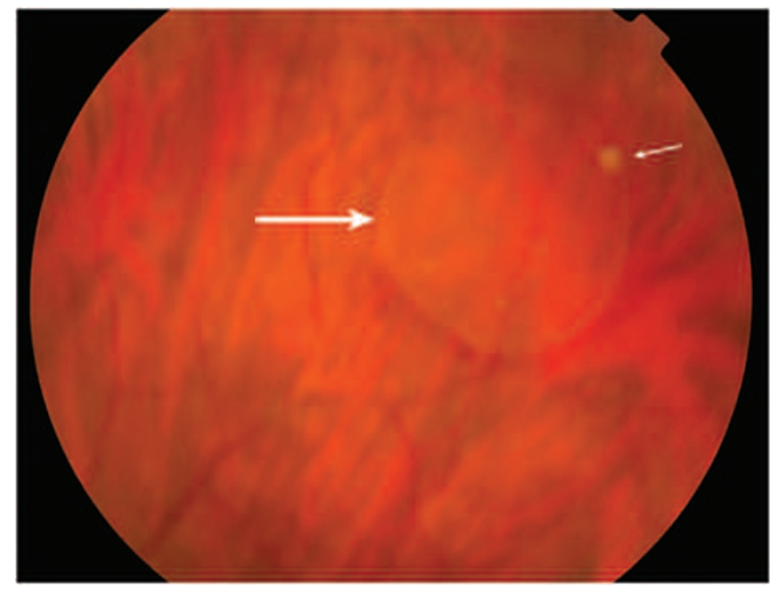

Figure 2 Colour photograph showing a vitreous cyst adjacent to an area of vascular sheathing (large arrow). Note the snowballs attached to the cyst's walls (small arrow). 


\section{Conflict of interest}

The authors declare no conflict of interest.

\section{References}

1 Cruciani F, Santino G, Salandri AG. Monolateral idiopathic cyst of the vitreous. Acta Ophthalmol Scand 1999; 77: 601-603.

2 Brewerton EW. Cysts in the vitreous. Trans Ophthalmol Soc UK 1913; 33: 93-94.

3 Hogan MJ, Zimmerman LE (eds). Ophthalmic Pathology, An Atlas Text, 2nd ed. Saunders: Philadelphia, 1962.

4 Pannarale C. A case of preretinal mobile cyst in a subject with congenital toxoplasmosis. G Ital Oftalmol 1964; 17: 306-317.

5 Bonfioli A, Damico F, Curi A, Orefice F. Intermediate uveitis. Sem Ophthalmol 2005; 20: 147-154.

PG Tranos ${ }^{1,2}$, P Ferrante $^{1}$ and C Pavesio ${ }^{1}$

${ }^{1}$ Moorfields Eye Hospital, London, UK

${ }^{2}$ Thessaloniki Retina Consultants, Thessaloniki, Greece

E-mail: ptranos@doctors.org.uk

Eye (2010) 24, 1115-1116; doi:10.1038/eye.2009.274; published online 13 November 2009

\section{Sir, Reply to Wertheim et al}

We read with great interest Wertheim et al's ${ }^{1}$ article on a new minim technique for diagnostic anterior chamber paracentesis. Although the use of a 25-gauge needle attached to a minim is an ingenious option in cases in which no better suited equipment is available, we would not wish readers to assume that this is the only, nor, in our opinion, the best, alternative to a specifically designed paracentesis pipette such as the $\mathrm{O}^{\prime}$ Rourke pipette. $^{2}$

Our group has previously described our technique for diagnostic anterior chamber paracentesis at the slit lamp. In that series of 70 procedures, 48 were performed with a 27-gauge needle pre-fixed to a $1 \mathrm{ml}$ insulin syringe (BD Medical, Oxford, UK), rather than a specifically designed pipette. This was found to be safe, with no serious complications using either instrument. ${ }^{3}$ Usage of the insulin syringe technique now predominates in our specialist service, with 56 of the 57 paracenteses performed by us in the last year utilizing this technique. As with the minim technique, the equipment required for the insulin syringe technique should be present in any ophthalmic department.

There are significant advantages of the insulin syringe technique that help make this procedure as safe as possible: a prefixed needle (with no risk of detachment or slippage), a measurable chamber (enabling monitoring of the volume withdrawn), and a slow predictable response to withdrawal of the plunger (vs a rapid fluid shift for a very small change of pressure on the bulb of a minim). The disadvantage of the insulin syringe technique is that, similar to the minim technique, it has a longer needle than the
$\mathrm{O}^{\prime}$ Rourke pipette $\left(\frac{1}{2}{ }^{\prime \prime}\right.$ for the insulin syringe vs $\frac{11}{4}$ for the O'Rourke). We do recognize that the insulin syringe technique is assisted by having an assistant to withdraw the plunger under the supervision of the operator, whereas this is not necessary with dedicated aqueous pipettes such as the O'Rourke or the minim technique.

Alongside welcoming the resourcefulness of the minim technique and recognizing its potential usefulness, we would propose that in general a specialist paracentesis pipette such as the $\mathrm{O}^{\prime}$ Rourke or a $1 \mathrm{ml}$ insulin syringe is likely to be the safer option.

\section{Conflict of interest}

The authors declare no conflict of interest.

\section{References}

1 Wertheim MS, Connell PP, Majid MA, Dick AD. The minim technique for diagnostic anterior chamber paracentesis. Eye 2009; 23(6): 1491.

2 O'Rourke J, Taylor DM, McDonald P, Kreutzer DL. An aqueous paracentesis pipet. Ophthalmic Surg 1991; 22(3): 166-167.

3 Cheung CMG, Durrani OM, Murray PI. The safety of anterior chamber paracentesis in patients with uveitis. Br J Ophthalmol 2004; 88(4): 582-583.

KN Amissah-Arthur, IJ Khan and AKO Denniston

Birmingham and Midland Eye Centre, City Hospital, Birmingham, West Midlands, UK E-mail: a.denniston@bham.ac.uk

Eye (2010) 24, 1116; doi:10.1038/eye.2009.285; published online 27 November 2009

Sir,

\section{Reply to Amissah-Arthur et al}

We thank Amissah-Arthur et al for commenting on our article on the minim technique for diagnostic paracentesis. We read with interest that they found it to be an ingenious technique. The minim technique is not proposed to replace other techniques of paracentesis, only to add to the possible techniques available. The main benefit of this technique is that the laboratory receives a specimen without any sharps and it avoids loss of specimen on collection. Another benefit is that only a single clinician is needed to perform the minim technique.

\section{Conflict of interest}

The authors declare no conflict of interest.

MS Wertheim, PP Connell, MA Majid and AD Dick

Department of Ophthalmology, Bristol Eye

Hospital, Bristol, Avon, UK

E-mail: drwertie@hotmail.com

Eye (2010) 24, 1116; doi:10.1038/eye.2009.286; published online 4 December 2009 